\title{
Adult-onset kaposiform hemangioendothelioma with neurofibromatosis type 1: A case report and literature review
}

\author{
Dong Hwi Kim ${ }^{1}$, Ji Sung Lee ${ }^{1}$, Jong Weon Shin ${ }^{2}$, Jean A Kim ${ }^{3}$, Young Joon Jun ${ }^{1}$ \\ ${ }^{1}$ Department of Plastic and Reconstructive Surgery, Bucheon St. Mary's Hospital, College of Medicine, The Catholic University of Korea, \\ Seoul; ${ }^{2}$ Department of Plastic and Reconstructive Surgery, Uijeongbu St. Mary's Hospital, College of Medicine, The Catholic University of \\ Korea, Seoul; ${ }^{3}$ Department of Clinical Pathology, Bucheon St. Mary's Hospital, College of Medicine, The Catholic University of Korea, Seoul, \\ Korea
}

Kaposiform hemangioendothelioma (KHE) is a very rare, locally aggressive vascular neoplasm. It occurs mostly in children and is rarely observed in adults. It typically originates on the skin, later affecting the deep soft tissue of the extremities, head or neck, and retroperitoneum by infiltrative growth. It is locally aggressive, does not regress spontaneously, and tends to metastasize locally as well as to the regional lymph nodes. In this article, we report a case of adult-onset KHE with neurofibromatosis type 1. The patient presented to our department with a 2-month history of a painful ulceration in her left popliteal area. Since KHE had not previously been reported in patients with neurofibromatosis, the diagnosis was difficult due to the similarity of the skin manifestation to neurofibromatosis-associated lesions. We share our experience of diagnosing and treating this rare case of adult-onset KHE.

Keywords Hemangioendothelioma / Neoplasms, vascular tissue / Neurofibromatoses / Skin manifestation / Neuralgia

Received: 12 Jun $2017 \bullet$ Revised: 17 Nov 2017 • Accepted: 1 Dec 2017

pISSN: 2234-6163 • elSSN: 2234-6171 • https://doi.org/10.5999/aps.2017.01032・Arch Plast Surg 2018;45:583-587
Correspondence: Young Joon Jun Department of Plastic and Reconstructive Surgery, Bucheon St. Mary's Hospital, College of Medicine, The Catholic University of Korea, 327 Sosa-ro, Wonmi-gu, Bucheon 14647 , Korea

Tel: $+82-32-340-7062$

Fax: +82-32-340-2544

E-mail: joony@catholic.ac.kr

\section{INTRODUCTION}

Kaposiform hemangioendothelioma (KHE) is a very rare, locally aggressive vascular neoplasm. It occurs mostly in children and is rarely observed in adults, with only approximately 26 adult KHE patients recorded [1]. KHE typically originates on the skin, later affecting the deep soft tissue of the extremities, head or neck, and retroperitoneum by infiltrative growth. It is locally aggressive, does not regress spontaneously, and tends to metastasize locally as well as to the regional lymph nodes. Cutaneous KHE typically appears as an erythematous violet-colored plaque with a firm consistency, involving the full thickness of the skin and extending into the subcutaneous tissue. Sometimes, the surface of the skin shows hemorrhagic areas as the earliest sign of the accompanying Kasabach-Merritt phenomenon [2] characterized by profound thrombocytopenia, lifethreatening hemorrhaging, and lymphangiomatosis [1].

Although any area of the body can be affected by KHE, the trunk and the proximal areas of the extremities are most commonly involved [2]. However, since KHE is rare, hemorrhagic and erythematous skin lesions are not the first parameters considered in the differential diagnosis of KHE. We present a rare case of KHE featuring underlying neurofibromatosis.

The study was approved by the Institutional Review Board of 
Bucheon St. Mary's Hospital (IRB No. HC17ZESI0056) and performed in accordance with the principles of the Declaration of Helsinki.

\section{CASE}

A 47-year-old woman presented to plastic and reconstructive surgery department with a 2-month history of a painful ulceration in her left popliteal area. She had suffered from neurofibromatosis type I as a child. Neurofibromatosis and café-au-lait lesions were scattered throughout the entire surface of her skin. A hyperpigmented area was also observed in the left inner thigh area. This was also thought to be a café-au-lait lesion caused by neurofibromatosis (Fig. 1A). She received a steroid injection and peripheral nerve block in the lesion to treat radiating pain in her left lower leg. She noted that an ulceration developed after the injection.

Physical examination revealed a skin ulceration measuring about $6.0 \times 3.5 \mathrm{~cm}$, with radiating pain in the area innervated by the left saphenous nerve (Fig. 1B). Magnetic resonance imaging (MRI) found multifocal cutaneous nodules and thickening of the skin and superficial soft tissue of the knee, which were related to cutaneous neurofibromatosis type 1 (Fig. 2A). We thought that some tumors associated with neurofibromatosis might have been affecting the saphenous nerve in the left lower leg, so excision of the ulcerative skin lesion and saphenous nerve decompression were performed to relieve the patient's symptoms. At the time of surgery, en bloc resection was performed including

Fig. 1. Preoperative findings

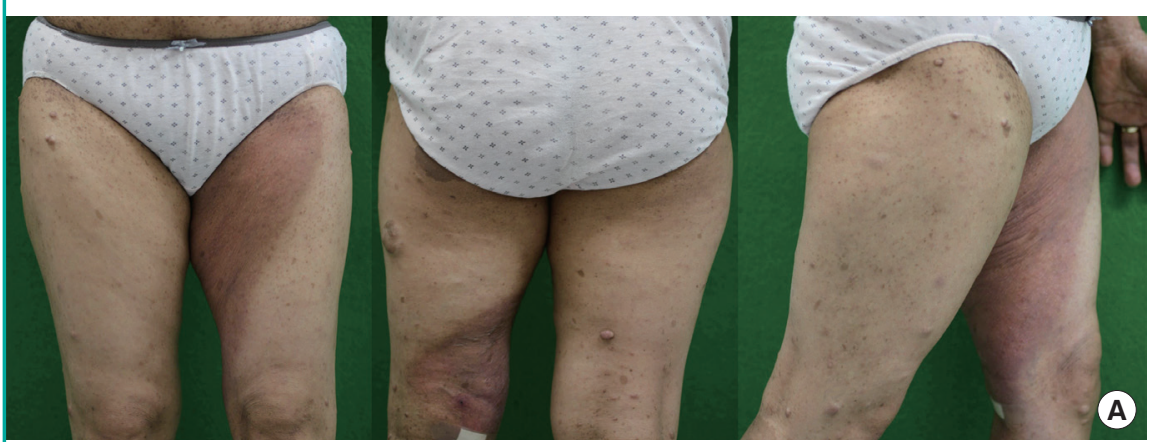

(A) Two months before surgery. A clinical photo shows what was thought to be a café-au-lait lesion in the left inner thigh area without skin ulceration. (B) A painful skin ulceration on the patient's left popliteal area is visible preoperatively. The lesion measured approximately $6.0 \times 3.5$ $\mathrm{cm}$, and was accompanied by radiating pain in the area innervated by the left saphenous nerve.

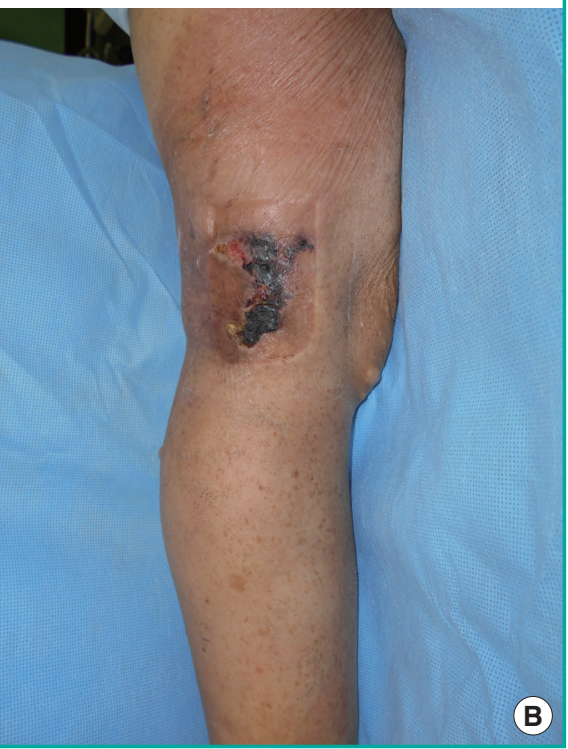

B

\section{Fig. 2. Magnetic resonance imaging scan}

(A) Multifocal cutaneous nodules and thickening of the skin and superficial soft tissue are present on the posteromedial aspect of the knee, which were related to cutaneous neurofibromatosis type 1 (red arrows). (B) There were localized areas of skin thickening and panniculitis-like lesions along the medial aspect of the thigh, raising the possibility of an intermediate-to-malignant vascular tumor (yellow arrows).
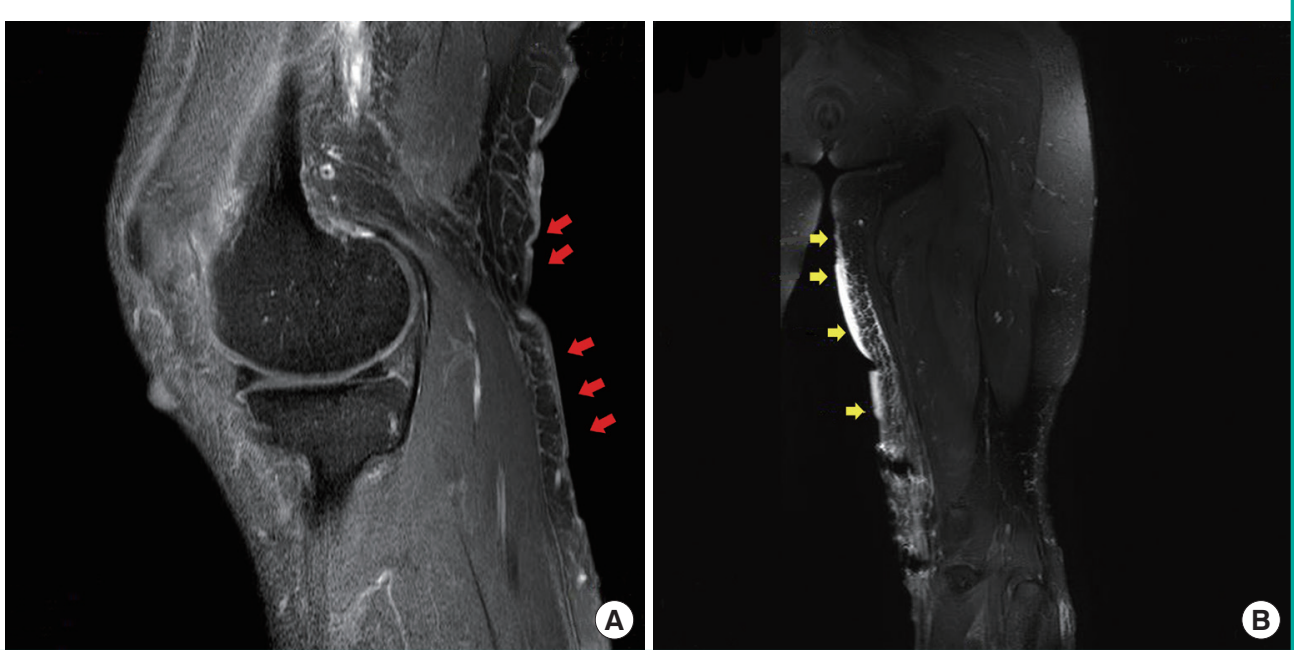
the entire ulcerative skin lesion. Mass-like clusters were evident around the saphenous nerve, and decompression was performed by removing them and releasing the vastoadductor membrane with sclerotic changes. After the operation, the painful radiation was somewhat alleviated. However, the pathologic results revealed KHE, contrary to our expectations, instead of neurofibroma.

Microscopically, the neoplasm was composed of infiltrating lobulated nodules of spindle-shaped endothelial cells separated by collagenous stroma that formed short fascicles and lined slitlike and round vessels. Immunohistochemical analysis revealed positive immunostaining for CD31 and CD34 in the capillarylining endothelial cells and arborizing epithelioid cells (Fig. 3). The absence of human herpesvirus- 8 in KHE underscores its different pathogenesis from Kaposi sarcoma [2,3].

Including coagulation time, the patient's laboratory findings were almost within normal ranges, except for a slightly elevated platelet count, and there was no history of bleeding diathesis. No findings suggesting lymphatic proliferation or the KasabachMerritt phenomenon were noted.

We planned a second operation for complete resection of the KHE. MRI was conducted before the operation to evaluate the extent of the tumor and to detect lymphangiectasia. MRI revealed localized skin thickening and a panniculitis-like lesion along the medial aspect of the thigh, raising the possibility of KHE (Fig. 2B). A $19 \times 12 \mathrm{~cm}$ erythematous lesion that was 0.7 $\mathrm{cm}$ in depth was found on her left leg, corresponding to the MRI findings. The suspicious area was marked for surgical excision. One month after the first operation, a wide excision (with a deep safety margin of about $1 \mathrm{~cm}$ ) with a split-thickness skin graft was performed. The pathologic results were the same as those of the first operation, and were confirmed as KHE. No

Fig. 3. Pathologic and immunohistochemical findings

(A) Photomicrographs showing a tumor with infiltrating lobulated nodules of spindle-shaped endothelial cells separated by collagenous stroma (yellow arrows) (hematoxylin and eosin [H\&E], $\times 40$ ). (B) Glomeruloid-like area with spindle-shaped endothelial cells composed of round small vessels surrounded by pericytes and hyaline globules (yellow circles) $\left(H \& E_{1} \times 400\right)$. (C) Photomicrographs of the tumor showing an immunohistochemical profile of diffuse positivity for $\operatorname{CD} 31(\times 400)$.
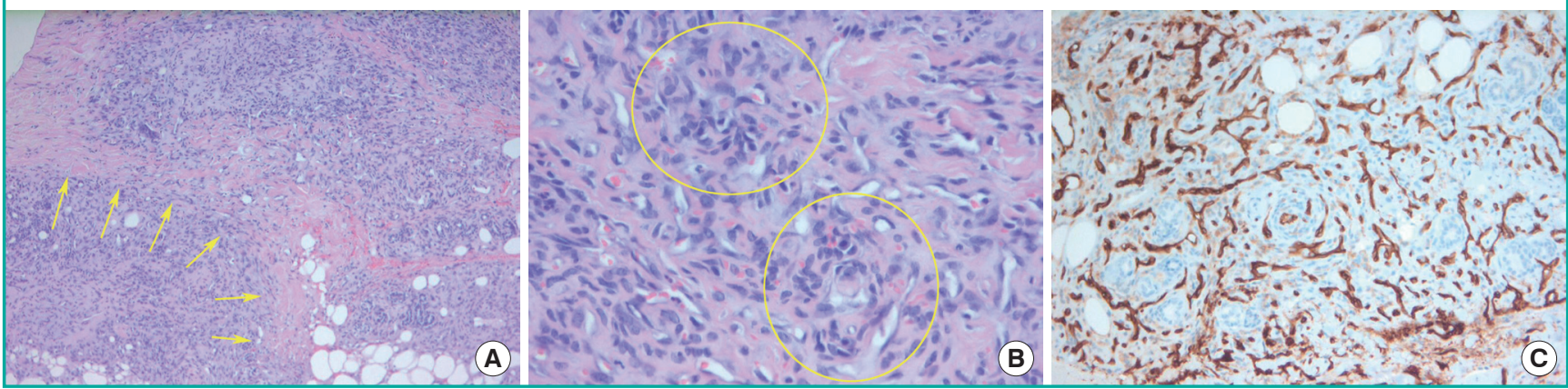

Fig. 4. Preoperative and postoperative findings

(A) An erythematous lesion measuring approximately $19 \times 12 \mathrm{~cm}$, including ulcerations on the patient's left leg, was marked with purple ink. (B) Wide excision was performed with a split-thickness skin graft. (C) Clinical photo taken 16 months postoperatively.
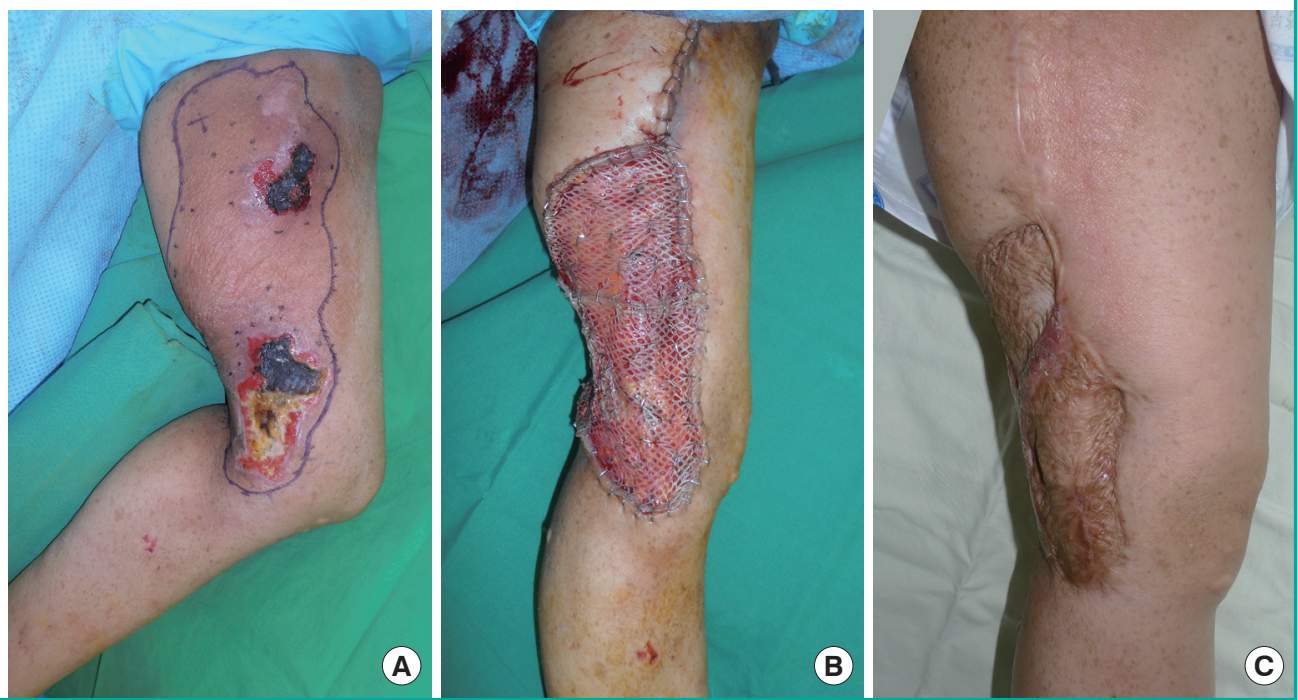
neurofibroma was found (Fig. 4). The patient was discharged after postoperative care. Postoperatively, follow-up visits continued for 2 years. The patient remained stable, without recurrence.

\section{DISCUSSION}

KHE is a rare disease that mainly occurs in children. If adult patients present with skin lesions, such as violet-colored papules and ulcerations, it is not easy to diagnose KHE at first. In a search of the PubMed database in 2016, only about 26 cases of adult KHE were found since the condition was first reported by Zukerberg et al. [4]. Several features of KHE in children are different from those seen in adults [5]. The most common locations of adult KHE are the extremities, torso, and the head and neck region. As in our case, no evidence of the Kasabach-Merritt phenomenon in adults has been reported [6].

Histologically, KHE consists of irregular tumor nodules that infiltrate the soft tissue and evoke a dense hyaline stromal response. Most tumors are intimately associated with thin-walled lymphatic vessels. These nodules contain glomeruloid nests of rounded or spindle-shaped epithelioid endothelial cells with abundant eosinophilic cytoplasm. The endothelial cells are arranged in cohesive nests with imperfect or partial lumen formation and only partially have a basal lamina [7]. Two important differential considerations in KHE are capillary (cellular) hemangioma and Kaposi sarcoma. Capillary hemangioma usually does not display spindling of the cells, and does not contain the signature glomeruloid structures of KHE. Moreover, unlike hemangioma, KHE must be followed very closely for local recurrence and growth. Kaposi sarcoma, on the other hand, is characterized by uniform spindling of the cells and often a striking inflammatory peripheral infiltrate. In contrast, KHE shows much greater variation from area to area. Human papillomavirus 8 has not been associated with KHE [6], and staining in the present case was negative as well.

The value of radiologic imaging, including computed tomography or ultrasonography, in patients with KHE is unclear because of the paucity of relevant reports [8,9]. In our case, MRI before surgery was considered appropriate given the patient's history of neurofibromatosis. However, after biopsy, KHE was a more appropriate finding. Both neurofibromatosis and KHE cause the involved skin and soft-tissue areas to show high-signal intensity on T2-weighted MRI images [10,11].

KHE is seldom diagnosed noninvasively, with a definitive diagnosis made based on histopathologic and immunohistochemical criteria. Nevertheless, MRI of the affected lesion may help to evaluate the evolution and tumoral extension of the lesion and facilitate the detection of lymphangiectasia. We performed MRI to define the necessary extent of excision more precisely [12].

In this case, it was difficult to suspect KHE clinically because the patient was an adult and the condition was accompanied by neurofibromatosis. In fact, we considered the brown- to purplecolored skin lesion to be a café-au-lait lesion caused by neurofibromatosis during the first visit. However, it was confirmed as KHE upon the later pathological diagnosis. Because a large caféau-lait macule has been reported in a neurofibromatosis patient [13], the pigmented area of skin in the inner thigh was thought to be related to the underlying disease of neurofibromatosis.

The patient received corticosteroid injections and nerve blockade continuously over a 1-year period to treat the pain of the skin lesion, so pain relief may have delayed the timing of her visit and diagnosis. In our literature review, we did not find any reports describing an association of cutaneous KHE with corticosteroid injections [6]. However, as KHE is a borderline malignancy, misdiagnosis may lead to invasion into surrounding structures, which has a mortality rate of up to $12 \%$ [14]. Fortunately, our patient only had locally aggressive vascular tumors without any metastasis, lymphatic proliferation, or the Kasabach-Merritt phenomenon, and a good prognosis was expected [4]. Still, the disease may have progressed if the diagnosis and treatment had been delayed further.

If we recognize the possibility of KHE in adult patients, physicians can be suspicious of KHE, which can enable a swift pathology- and radiology-based diagnosis and prompt the provision of proper treatment when encountering a patient who presents with an increasingly vascularized skin lesion. An improved prognosis can be anticipated if a patient's coagulation status is examined and other lymphangitic lesions are confirmed.

Because KHE is rare, no treatment guidelines have been established. Early and complete surgical treatment is the most effective way to treat KHE, especially when it is associated with the Kasabach-Merritt phenomenon. Complete surgical removal is the primary therapeutic option, and most tumors in adults can be successfully excised. However, systemic treatment should be directed toward attributable systemic diseases and lesions not amenable to resection. Several nonsurgical treatments, alone or in combination, have been used to manage KHE successfully. These include interferon- $\alpha$; a chemotherapeutic regimen consisting of actinomycin D, cyclophosphamide, and vincristine; ticlopidine hydrochloride with aspirin; corticosteroids; radiotherapy; and embolization. However, a standardized treatment protocol has not yet been established owing to the rarity of this disease [15]. If the tumor recurs, additional medical treatment 
should be considered because KHE responds well to radiation, chemotherapy, and corticosteroid therapy.

We report a rare case of adult-onset KHE. To our knowledge, patients with underlying neurofibromatosis are the only patients who experience KHE. However, due to the rarity of KHE, the link between neurofibromatosis and KHE incidence has been difficult to establish. Adult-onset KHE is a very rare disease that is susceptible to delayed diagnosis. However, if erythematous violet-colored skin lesions are observed that do not show consistent improvement, we should not overlook KHE in the differential diagnosis.

\section{NOTES}

\section{Conflict of interest}

No potential conflict of interest relevant to this article was reported.

\section{Ethical approval}

The study was approved by the Institutional Review Board of Bucheon St. Mary's Hospital (IRB No. HC17ZESI0056) and performed in accordance with the principles of the Declaration of Helsinki. Written informed consent was obtained.

\section{Patient consent}

The patient provided written informed consent for the publication and the use of her images.

\section{REFERENCES}

1. Fernandez Y, Bernabeu-Wittel M, Garcia-Morillo JS. Kaposiform hemangioendothelioma. Eur J Intern Med 2009;20: 106-13.

2. Requena L, Kutzner H. Hemangioendothelioma. Semin Diagn Pathol 2013;30:29-44.

3. Lyons LL, North PE, Mac-Moune Lai F, et al. Kaposiform hemangioendothelioma: a study of 33 cases emphasizing its pathologic, immunophenotypic, and biologic uniqueness from juvenile hemangioma. Am J Surg Pathol 2004;28:559-
68.

4. Zukerberg LR, Nickoloff BJ, Weiss SW. Kaposiform hemangioendothelioma of infancy and childhood: an aggressive neoplasm associated with Kasabach-Merritt syndrome and lymphangiomatosis. Am J Surg Pathol 1993;17:321-8.

5. Karnes JC, Lee BT, Phung T, et al. Adult-onset kaposiform hemangioendothelioma in a posttraumatic site. Ann Plast Surg 2009;62:456-8.

6. Vashi P, Abboud E, Bier-Laning C, et al. Adult-onset Kaposiform hemangioendothelioma of the tongue: case report and review of the literature. Curr Oncol 2016;23:e517-20.

7. Weiss SW, Goldblum JR, Enzinger FM. Enzinger and Weiss's soft tissue tumors. 4th ed. St. Louis: Mosby; 2001.

8. Vogel AM, Alesbury JM, Fox VL, et al. Complex pancreatic vascular anomalies in children. J Pediatr Surg 2006;41:4738

9. Rubinowitz AN, Moreira AL, Naidich DP. Mediastinal hemangioendothelioma: radiologic-pathologic correlation. J Comput Assist Tomogr 2000;24:721-3.

10. Croteau SE, Liang MG, Kozakewich HP, et al. Kaposiform hemangioendothelioma: atypical features and risks of Kasabach-Merritt phenomenon in 107 referrals. J Pediatr 2013; 162:142-7.

11. Hassell DS, Bancroft LW, Kransdorf MJ, et al. Imaging appearance of diffuse neurofibroma. AJR Am J Roentgenol 2008; 190:582-8.

12. Cooper JG, Edwards SL, Holmes JD. Kaposiform haemangioendothelioma: case report and review of the literature. $\mathrm{Br}$ J Plast Surg 2002;55:163-5.

13. Yang CC, Happle R, Chao SC, et al. Giant café-au-lait macule in neurofibromatosis 1: a type 2 segmental manifestation of neurofibromatosis 1? J Am Acad Dermatol 2008;58: 493-7.

14. el-Dessouky M, Azmy AF, Raine PA, et al. Kasabach-Merritt syndrome. J Pediatr Surg 1988;23:109-11.

15. Enjolras O, Wassef M, Mazoyer E, et al. Infants with Kasabach-Merritt syndrome do not have "true" hemangiomas. J Pediatr 1997;130:631-40. 